\title{
Generalized Inferences about the Mean Vector of Several Multivariate Gaussian Processes
}

\author{
Pilar Ibarrola ${ }^{1}$ and Ricardo Vélez ${ }^{2}$ \\ ${ }^{1}$ Statistics Department, Universidad Complutense de Madrid, 28040 Madrid, Spain \\ ${ }^{2}$ Statistics Department, UNED, 28040 Madrid, Spain \\ Correspondence should be addressed to Pilar Ibarrola; ibarrola@mat.ucm.es
}

Received 22 June 2015; Accepted 7 October 2015

Academic Editor: Dejian Lai

Copyright (C) 2015 P. Ibarrola and R. Vélez. This is an open access article distributed under the Creative Commons Attribution License, which permits unrestricted use, distribution, and reproduction in any medium, provided the original work is properly cited.

\begin{abstract}
We consider in this paper the problem of comparing the means of several multivariate Gaussian processes. It is assumed that the means depend linearly on an unknown vector parameter $\theta$ and that nuisance parameters appear in the covariance matrices. More precisely, we deal with the problem of testing hypotheses, as well as obtaining confidence regions for $\theta$. Both methods will be based on the concepts of generalized $p$ value and generalized confidence region adapted to our context.
\end{abstract}

\section{Introduction}

The generalized $p$ values to test statistical hypotheses in the presence of nuisance parameters are introduced by Tsui and Weerahandi (1989) [1], where the univariate Behrens-Fisher problem, as well as other examples, is considered in order to illustrate the usefulness of this approach. Afterwards Weerahandi (1993) [2] introduces the generalized confidence intervals.

In 2004, Gamage et al. [3] developed a procedure based on the generalized $p$ values to test the equality of the mean vectors of two multivariate normal populations with different covariance matrices. They also construct a confidence region for the means difference, using the concept of generalized confidence regions. Finally, by means of the generalized $p$ value approach, a solution is obtained for the heteroscedastic MANOVA problem, but without reaching the desirable invariance property.

In 2007, Lin et al. [4] considered the generalized inferences on the common mean vector of several multivariate normal populations. They obtained a confidence region for the common mean vector and simultaneous confidence intervals for its components. Their method is numerically compared with other existing methods, with respect to the expected area and coverage probabilities.
In 2008, $\mathrm{Xu}$ and Wang [5] considered the problem of comparing the means of $K$ populations with heteroscedastic variances. They provided a new generalized $p$ value procedure for testing the equality of means, assuming that the variables are univariate and normally distributed. Numerical results show that their generalized $p$ value test works better than a generalized $F$-test. We will set out our MANOVA problem as a generalization of their framework.

In 2012, Zhang [6] considered the general linear hypothesis testing (GLHT) in an heteroscedastic one-way MANOVA. The multivariate Behrens-Fisher problem is a special case of GLHT.

In this paper we first consider the generalized inference for the case of two continuous time Gaussian processes. Later, the results will be extended for such $N$ processes. In both cases, for the testing problem, the main step is constructing a generalized test process and analyzing the associated generalized $p$ value, proving some linear invariance properties.

With respect to the construction of generalized confidence regions, one should use a generalized pivotal quantity and use the approach of multiple comparisons as in [4].

Finally, in the same line of Zhang [6], we consider the general linear hypothesis testing (GLHT) as a generalization of the MANOVA, adapting the setting and method of this paper. 
It must be emphasized that all the references above develop these techniques for discrete univariate or multivariate models, whereas here we are concerned with a continuous time model. It is well known that when the underlying phenomenon is in essence continuous, even if it is observed at a sequence of epochs $n \Delta t$, different models may be necessary for distinct values of $\Delta t$. On the contrary a continuous time model embodies simultaneously all the statistical properties of the time series obtained for each value of $\Delta t$.

\section{Continuous Time Generalized Tests and Confidence Regions}

Let $\left\{X_{t}\right\}_{t \in T}$ be a $k$-dimensional stochastic process with distribution depending on the unknown parameter $\xi=(\theta, \zeta)$, $\theta$ being the vector of parameters of interest and $\zeta$ a nuisance parameter vector. For any random vector $Y, \widetilde{Y}$ will denote its observed value.

For the problem of testing a null hypothesis $H_{0}: \theta \leq \theta_{0}$ against the alternative $H_{1}: \theta>\theta_{0}$, where $\theta_{0}$ is a given vector (the inequalities like $\theta_{1} \leq \theta_{2}$ should be understood componentwise.), a generalized test process is defined, following [3], as follows.

Definition 1. A generalized test process $W_{t}(X, \widetilde{X}, \theta, \zeta)$ is, for each $t \in T$, a one-dimensional function depending on $\left\{X_{s}\right\}_{s \leq t}$ and its observed value $\left\{\widetilde{X}_{s}\right\}_{s \leq t}$, as well as the parameter value $\xi=(\theta, \zeta)$, satisfying the following:

(1) the distribution of $W_{t}\left(X, \widetilde{X}, \theta_{0}, \zeta\right)$ does not depend on $\zeta$, for any fixed $\widetilde{X}$,

(2) the observed value $\widetilde{W_{t}}=W_{t}\left(\widetilde{X}, \widetilde{X}, \theta_{0}, \zeta\right)$ does not depend on $\zeta$,

(3) $P_{\theta}\left\{W_{t}(X, \widetilde{X}, \theta, \zeta) \geq w\right\}$ is nondecreasing in every component of $\theta$, for any $w \in \mathbb{R}$ and any fixed $\widetilde{X}$ and $\zeta$.

Under the above conditions, the generalized $p$ value is defined as

$$
\begin{aligned}
P_{t} & =\sup _{\theta \leq \theta_{0}} P_{\theta}\left\{W_{t}(X, \widetilde{X}, \theta, \zeta) \geq \widetilde{W}_{t}\right\} \\
& =P_{\theta_{0}}\left\{W_{t}\left(X, \widetilde{X}, \theta_{0}, \zeta\right) \geq \widetilde{W}_{t}\right\} .
\end{aligned}
$$

When testing $H_{0}: \theta=\theta_{0}$ versus $H_{1}: \theta \neq \theta_{0}$, condition (3) must be replaced by

$\left(3^{\prime}\right) W_{t}(X, \widetilde{X}, \theta, \zeta)$ is stochastically larger under $H_{1}$ than under $H_{0}$, for any fixed $\widetilde{X}$ and $\zeta$.

In this case, the generalized $p$ value is given by

$$
p_{t}=P_{\theta_{0}}\left\{W_{t}\left(X, \widetilde{X}, \theta_{0}, \zeta\right) \geq \widetilde{W}_{t}\right\}
$$

Towards the confidential estimation of $\theta$, we give the following definition.
Definition 2. A generalized pivotal quantity $W_{t}(X, \widetilde{X}, \theta, \zeta)$ is, for each $t \in T$, a one-dimensional function satisfying the following:

(1) the distribution of $W_{t}(X, \widetilde{X}, \theta, \zeta)$ does not depend on $\theta$ nor $\zeta$,

(2) the observed value $\widetilde{W_{t}}=W_{t}(\widetilde{X}, \widetilde{X}, \theta, \zeta)$ does not depend on $\zeta$.

Then, if $c_{1}<c_{2}$ are such that

$$
P_{\theta}\left\{c_{1} \leq W_{t}(X, \widetilde{X}, \theta, \zeta) \leq c_{2}\right\}=1-\gamma
$$

$\left\{\theta \mid c_{1} \leq \widetilde{W_{t}} \leq c_{2}\right\}$ is a $100(1-\gamma) \%$ generalized confidence region for $\theta$.

\section{Estimation Method}

In the previous paper [7] we have considered the confidence estimation of a $k$-dimensional parameter $\theta$, when observing a continuous time $p$-dimensional Gaussian process $X_{t}$, with covariances function $B\left(t, t^{\prime}\right)=\sigma^{2} K\left(t, t^{\prime}\right)$ and mean function $\mu(t)=A(t) \theta$, where $K\left(t, t^{\prime}\right)$ and $A(t)$ are known matrices, but $\sigma^{2}$ and $\theta$ are unknown parameters. More concrete assumptions were specified by Ibarrola and Vélez [7] and we will here suppose that they hold for all the considered processes.

The estimation method of $\theta$, described in [7], is based on the estimator

$$
\theta_{t}=\Sigma_{t}^{-1} \widehat{\theta}_{t} \quad \text { with } \widehat{\theta}_{t}=\int_{0}^{t} \widehat{f}_{t}^{\prime}(u) X_{u} d u,
$$

where $\widehat{f}_{t}^{\prime}$ is the $k \times p$-matrix with columns in $L^{2, p}[0, t]$ satisfying the equation

$$
A(u)=\int_{0}^{t}\left[K\left(u, u^{\prime}\right)+A(u) A^{\prime}\left(u^{\prime}\right)\right] \hat{f}_{t}\left(u^{\prime}\right) d u^{\prime},
$$

$(\forall u \leq t)$

and $\Sigma_{t}$ is given by

$$
\Sigma_{t}=\int_{0}^{t} \widehat{f}_{t}^{\prime}(u) A(u) d u .
$$

As proved in [7], $\left\{\theta_{t}\right\}$ does not depend on $\sigma^{2}$ and constitutes a Gaussian process such that

$$
\begin{aligned}
\mathrm{E}_{\theta}\left[\theta_{t}\right] & =\theta, \\
\operatorname{Cov}_{\theta}\left(\theta_{t}, \theta_{t^{\prime}}\right) & =\sigma^{2} \sigma_{t \vee t^{\prime}} \quad \text { where } \sigma_{t}=\Sigma_{t}^{-1}-I .
\end{aligned}
$$

Consequently, $\theta_{t}$ and $\theta_{t_{2}^{\prime}}-\theta_{t_{1}^{\prime}}$ are independent if $t_{1}^{\prime}<t_{2}^{\prime} \leq t$, while

$$
\begin{array}{r}
\mathrm{E}_{\theta}\left[\left(\theta_{t^{\prime}}-\theta\right)\left(\theta_{t_{2}}-\theta_{t_{1}}\right)^{\prime}\right]=\sigma^{2}\left(\sigma_{t_{2}}-\sigma_{t_{1}}\right) \\
\text { if } t^{\prime} \leq t_{1}<t_{2} .
\end{array}
$$

Moreover, $\theta_{t}$ is a mean square consistent estimator of $\theta$, since all the eigenvalues of the covariance matrix $\sigma_{t}$ converge to 0 . 
In order to estimate $\sigma^{2}$, if $t_{1}<t_{2}$ are arbitrarily chosen so that $\sigma_{t_{1}}-\sigma_{t_{2}}$ is nonsingular, we can consider the random variable

$$
\eta=\left(\theta_{t_{2}}-\theta_{t_{1}}\right)^{\prime}\left(\sigma_{t_{1}}-\sigma_{t_{2}}\right)^{-1}\left(\theta_{t_{2}}-\theta_{t_{1}}\right)
$$

such that $\eta / \sigma^{2}$ has a $\chi_{k}^{2}$ distribution.

\section{The Behrens-Fisher Problem}

We first consider the case of two independent Gaussian stochastic processes, $\left\{X_{t}=\left(X_{t}^{1}, \ldots, X_{t}^{p}\right)^{\prime}\right\}$ and $\left\{Y_{s}=\right.$ $\left.\left(Y_{s}^{1}, \ldots, Y_{s}^{q}\right)^{\prime}\right\}$, of dimensions $p$ and $q$, respectively, and with similar characteristics. More precisely,

$$
\begin{aligned}
\mathrm{E}_{\theta_{1}}\left[X_{t}\right] & =A_{1}(t) \theta_{1}, \\
\operatorname{Cov}\left(X_{t}, X_{t^{\prime}}\right) & =\sigma_{1}^{2} K_{1}\left(t, t^{\prime}\right), \\
\mathrm{E}_{\theta_{2}}\left[Y_{s}\right] & =A_{2}(s) \theta_{2}, \\
\operatorname{Cov}\left(Y_{s}, Y_{s^{\prime}}\right) & =\sigma_{2}^{2} K_{2}\left(s, s^{\prime}\right),
\end{aligned}
$$

where $\theta_{1}, \theta_{2} \in \mathbb{R}^{k}$ and $\sigma_{1}^{2}, \sigma_{2}^{2}$ are unknown parameters, while $A_{1}(t), A_{2}(s)$ and $K_{1}\left(t, t^{\prime}\right), K_{2}\left(s, s^{\prime}\right)$ are given matrices of appropriate dimensions.

We will focus on the Behrens-Fisher type problem of comparing the parameters $\theta_{1}$ and $\theta_{2}$ and, more concretely, our aim is to make inferences about $\theta_{1}-\theta_{2}$, based on the progressive observation of both processes $X_{t}$ and $Y_{s}$.

The progressive estimators $\theta_{t}^{(1)}$ and $\theta_{t}^{(2)}$ of $\theta_{1}$ and $\theta_{2}$ can be constructed according to (4), (5), and (6). For $i=1,2, \Sigma_{t}^{(i)}$ and $\sigma_{t}^{(i)}$ will denote the characteristics defined in (6), (7) for $\left\{X_{t}\right\}$ and $\left\{Y_{s}\right\}$, respectively. We thus obtain the unbiased estimator of $\delta=\theta_{1}-\theta_{2}$ :

$$
T_{t, s}=\theta_{t}^{(1)}-\theta_{s}^{(2)}
$$

which is normally distributed, satisfies $\mathrm{E}\left[T_{t, s}\right]=\delta$, and, since $\left\{\theta_{t}^{(1)}\right\}$ and $\left\{\theta_{s}^{(2)}\right\}$ are independent, has covariance

$$
\operatorname{Cov}\left(T_{t, s}, T_{t^{\prime}, s^{\prime}}\right)=\sigma_{1}^{2} \sigma_{t \vee t^{\prime}}^{(1)}+\sigma_{2}^{2} \sigma_{s \vee s^{\prime}}^{(2)}
$$

In order to estimate $\sigma_{1}^{2}$ and $\sigma_{2}^{2}$, we can take

$$
\begin{aligned}
& \eta_{1}=\left(\theta_{t_{2}}^{(1)}-\theta_{t_{1}}^{(1)}\right)^{\prime}\left(\sigma_{t_{1}}^{(1)}-\sigma_{t_{2}}^{(1)}\right)^{-1}\left(\theta_{t_{2}}^{(1)}-\theta_{t_{1}}^{(1)}\right), \\
& \eta_{2}=\left(\theta_{s_{2}}^{(2)}-\theta_{s_{1}}^{(2)}\right)^{\prime}\left(\sigma_{s_{1}}^{(2)}-\sigma_{s_{2}}^{(2)}\right)^{-1}\left(\theta_{s_{2}}^{(2)}-\theta_{s_{1}}^{(2)}\right),
\end{aligned}
$$

where $t_{1}<t_{2}$ and $s_{1}<s_{2}$ are such that $\sigma_{t_{1}}^{(1)}-\sigma_{t_{2}}^{(1)}$ and $\sigma_{s_{1}}^{(2)}-\sigma_{s_{2}}^{(2)}$ are nonsingular. In this way $\eta_{1} / \sigma_{1}^{2}$ and $\eta_{2} / \sigma_{2}^{2}$ are independent, $\chi_{k}^{2}$-distributed random variables, independent of $T_{t, s}$.

4.1. Generalized $p$ Value for the Behrens-Fisher Problem. We consider the problem of testing $H_{0}: \delta=0$ against the alternative $H_{1}: \delta \neq 0$ and we look for a generalized test process $W_{t, s}\left(\theta_{t}^{(1)}, \theta_{s}^{(2)}, \widetilde{\theta}_{t}^{(1)}, \widetilde{\theta}_{s}^{(2)}, \delta, \sigma_{1}^{2}, \sigma_{2}^{2}\right)$.
The covariance matrix of $T_{t, s}$ is

$$
U_{t, s}=\sigma_{1}^{2} \sigma_{t}^{(1)}+\sigma_{2}^{2} \sigma_{s}^{(2)}
$$

and can be estimated by means of

$$
V_{t, s}=\eta_{1} \sigma_{t}^{(1)}+\eta_{2} \sigma_{s}^{(2)}
$$

Recall that $\widetilde{T}_{t, s}, \widetilde{\eta}_{i}$, and $\widetilde{V}_{t, s}$ represent the observed values, obtained when $\theta_{t}^{(1)}$ and $\theta_{s}^{(2)}$ are replaced with $\widetilde{\theta}_{t}^{(1)}$ and $\widetilde{\theta}_{s}^{(2)}$, and let us define

$$
\begin{aligned}
d_{t, s} & =\widetilde{V}_{t, s}^{-1 / 2} T_{t, s}, \\
Z_{t, s} & =\left(\widetilde{V}_{t, s}^{-1 / 2} U_{t, s} \widetilde{V}_{t, s}^{-1 / 2}\right)^{-1 / 2} d_{t, s} .
\end{aligned}
$$

Under $H_{0}, d_{t, s}$ has distribution $N_{k}\left(0, \widetilde{V}_{t, s}^{-1 / 2} U_{t, s} \widetilde{V}_{t, s}^{-1 / 2}\right)$, so that the distribution of $Z_{t, s}$ is $N_{k}(0, I)$. Moreover

$$
R_{t, s}=\widetilde{V}_{t, s}^{-1 / 2}\left(\sigma_{1}^{2} \sigma_{t}^{(1)} \frac{\widetilde{\eta}_{1}}{\eta_{1}}+\sigma_{2}^{2} \sigma_{s}^{(2)} \frac{\widetilde{\eta}_{2}}{\eta_{2}}\right) \widetilde{V}_{t, s}^{-1 / 2}
$$

does not depend on $\delta$ and its distribution is independent of the parameters, since $u_{1}=\eta_{1} / \sigma_{1}^{2}$ and $u_{2}=\eta_{2} / \sigma_{2}^{2}$ are independent, $\chi_{k}^{2}$ distributed random variables, independent of $Z_{t, s}$. The observed value of $R_{t, s}$ is $\widetilde{R}_{t, s}=\widetilde{V}_{t, s}^{-1 / 2} U_{t, s} \widetilde{V}_{t, s}^{-1 / 2}$. Finally,

$$
\begin{aligned}
W_{t, s} & =Z_{t, s}^{\prime} R_{t, s} Z_{t, s} \\
& =Z_{t, s}^{\prime} \widetilde{V}_{t, s}^{-1 / 2}\left(\frac{\widetilde{\eta}_{1}}{u_{1}} \sigma_{t}^{(1)}+\frac{\widetilde{\eta}_{2}}{u_{2}} \sigma_{s}^{(2)}\right) \widetilde{V}_{t, s}^{-1 / 2} Z_{t, s}
\end{aligned}
$$

is a one-dimensional random variable with distribution not depending on $\left(\sigma_{1}^{2}, \sigma_{2}^{2}\right)$, whose observed value is $\widetilde{W}_{t, s}=$ $\widetilde{T}_{t, s}^{\prime} \widetilde{V}_{t, s}^{-1} \widetilde{T}_{t, s}$, that neither depends on $\left(\sigma_{1}^{2}, \sigma_{2}^{2}\right)$.

Under $H_{1}$, when $\delta \neq 0$, as $T_{t, s}$ has distribution $N_{k}\left(\delta, U_{t, s}\right)$, the distribution of $Z_{t, s}$ is $N_{k}(\bar{\delta}, I)$, with $\bar{\delta}=$ $\left(\widetilde{V}_{t, s}^{-1 / 2} U_{t, s} \widetilde{V}_{t, s}^{-1 / 2}\right)^{-1 / 2} \widetilde{V}_{t, s}^{-1 / 2} \delta$. Note that, given $u_{1}$ and $u_{2}$, since $R_{t, s}$ is a positive definite matrix, $W_{t, s}$ is stochastically larger under $H_{1}$ than under $H_{0}$. So, since the conditions (1), (2), and (3) of Definition 1 are verified, we have proved the following result.

Proposition 3. $W_{t, s}$ is a generalized test process for testing $H_{0}$ : $\delta=0$ against $H_{1}: \delta \neq 0$.

In order to simplify the expression of $W_{t, s}$, we will put

$$
\begin{aligned}
& M_{1}=M_{1}(t, s)=\widetilde{V}_{t, s}^{-1 / 2} \widetilde{\eta}_{1} \sigma_{t}^{(1)} \widetilde{V}_{t, s}^{-1 / 2}, \\
& M_{2}=M_{2}(t, s)=\widetilde{V}_{t, s}^{-1 / 2} \widetilde{\eta}_{2} \sigma_{s}^{(2)} \widetilde{V}_{t, s}^{-1 / 2}
\end{aligned}
$$

which are positive definite matrices such that $M_{1}+M_{2}=I$. Then

$$
\begin{aligned}
W_{t, s} & =Z_{t, s}^{\prime}\left(\frac{M_{1}}{u_{1}}+\frac{M_{2}}{u_{2}}\right) Z_{t, s} \\
& =\frac{1}{2 k} \bar{Z}_{t, s}^{\prime}\left(\frac{M_{1}}{\beta}+\frac{I-M_{1}}{1-\beta}\right) \bar{Z}_{t, s},
\end{aligned}
$$


where, under $H_{0}, \bar{Z}_{t, s}=\sqrt{2 k} Z_{t, s} /\left(u_{1}+u_{2}\right)^{1 / 2}$ has a $k$ multivariate Student's $t$-distribution with $2 k$ degrees of freedom and $\beta=u_{1} /\left(u_{1}+u_{2}\right)$ is a random variable with distribution $\operatorname{Beta}(k / 2, k / 2)$, which is independent of $\bar{Z}_{t, s}$.

Thus the generalized $p$ value of the given test:

$$
\begin{aligned}
p_{t, s} & \left(M_{1}\right)=P_{\delta=0}\left\{W_{t, s} \geq \widetilde{W}_{t, s}\right\} \\
= & P_{\delta=0}\left\{\bar{Z}_{t, s}^{\prime}\left(\frac{M_{1}}{\beta}+\frac{I-M_{1}}{1-\beta}\right) \bar{Z}_{t, s} \geq 2 k \widetilde{W}_{t, s}\right\}
\end{aligned}
$$

may be calculated once $M_{1}$ is observed.

4.2. Invariance Properties of the Generalized $p$ Value. Let us assume that the basic processes are transformed by means of

$$
\begin{aligned}
& X_{t}^{\star}=Q_{1} X_{t}, \\
& Y_{s}^{\star}=Q_{2} Y_{s},
\end{aligned}
$$

where $Q_{1}, Q_{2}$ are nonsingular square matrices of order $p$ and $q$, respectively. The characteristics of the transformed processes are

$$
\begin{aligned}
\mathrm{E}_{\theta_{1}}\left[X_{t}^{\star}\right] & =A_{1}^{\star}(t) \theta_{1}, \\
\mathrm{E}_{\theta_{2}}\left[Y_{t}^{\star}\right] & =A_{2}^{\star}(s) \theta_{2}, \\
\operatorname{Cov}\left(X_{t}^{\star}, X_{t^{\prime}}^{\star}\right) & =\sigma_{1}^{2} K_{1}^{\star}\left(t, t^{\prime}\right), \\
\operatorname{Cov}\left(Y_{s}^{\star}, Y_{s^{\prime}}^{\star}\right) & =\sigma_{2}^{2} K_{2}^{\star}\left(s, s^{\prime}\right),
\end{aligned}
$$

where $A_{i}^{\star}(\cdot)=Q_{i} A_{i}(\cdot)$ and $K_{i}^{\star}(\cdot, \cdot)=Q_{i} K_{i}(\cdot, \cdot) Q_{i}^{\prime}$.

For $X_{t}^{\star}$, the solution $\widehat{f}_{t}^{\star}(u)$ of (5) with the new characteristics will be related to $\widehat{f}_{t}(u)$ by means of $\widehat{f}_{t}^{\star}(u)=\left(Q_{1}^{\prime}\right)^{-1} \widehat{f}_{t}(u)$. Therefore, according to (4) and (6), we get

$$
\begin{aligned}
& \widehat{\theta}_{t}^{(1) \star}=\widehat{\theta}_{t}^{(1)}, \\
& \Sigma_{t}^{(1) \star}=\Sigma_{t}^{(1)},
\end{aligned}
$$

so that $\theta_{t}^{(1) \star}=\theta_{t}^{(1)}, \sigma_{t}^{(1) \star}=\sigma_{t}^{(1)}$, and $\eta_{1}^{\star}=\eta_{1}$.

The same results hold for $Y_{s}^{\star}$ yielding $T_{t, s}^{\star}=T_{t, s}$, as well as $W_{t, s}^{\star}=W_{t, s}$. In this sense the generalized test process and the corresponding generalized $p$ value are invariant under the proposed transformations.

4.3. Generalized Confidence Region for $\delta$. For any value of the unknown parameter $\delta$, the difference $T_{t, s}^{\delta}=T_{t, s}-\delta$ has distribution $N_{k}\left(0, U_{t, s}\right)$ and $Z_{t, s}^{\delta}=\left(\widetilde{V}_{t, s}^{-1 / 2} U_{t, s} \widetilde{V}_{t, s}^{-1 / 2}\right)^{-1 / 2} \widetilde{V}_{t, s}^{-1 / 2} T_{t, s}^{\delta}$ is $N_{k}(0, I)$. Hence

$$
W_{t, s}^{\delta}=\left(Z_{t, s}^{\delta}\right)^{\prime} R_{t, s} Z_{t, s}^{\delta}
$$

has the same distribution as $W_{t, s}$ under $H_{0}: \delta=0$, which is independent of all the parameters, while its observed value $\widetilde{W}_{t, s}^{\delta}$ does not depend on the nuisance parameters $\left(\sigma_{1}^{2}, \sigma_{2}^{2}\right)$. Since conditions (1) and (2) of Definition 2 are accomplished, the following result is established.
Proposition 4. $W_{t, s}^{\delta}$ is a generalized pivotal quantity and $\{\delta \in$ $\left.\mathbb{R}^{k} \mid \widetilde{W}_{t, s}^{\delta} \leq c_{\gamma}\right\}$ is a generalized confidence region for $\delta$, whenever $P\left\{W_{t, s}^{\delta} \leq c_{\gamma}\right\}=1-\gamma$.

According to (20), once $M_{1}$ is observed, the constant $c_{\gamma}$ may be determined and the confidence region for $\delta$ is the ellipsoid in $\mathbb{R}^{k}$ :

$$
\mathscr{R}=\left\{\delta \in \mathbb{R}^{k} \mid\left(\delta-\widetilde{T}_{t, s}\right)^{\prime} \widetilde{V}_{t, s}^{-1}\left(\delta-\widetilde{T}_{t, s}\right) \leq c_{\gamma}\right\}
$$

which is centered at $\widetilde{T}_{t, s}$ and with axes in the direction of each eigenvector of $\widetilde{V}_{t, s}$ of length $\pm \sqrt{c_{\gamma} \lambda}$, where $\lambda$ is the corresponding eigenvalue. Thus the $k$-dimensional volume of the confidence region results in

$$
\frac{\left(\pi c_{\gamma}\right)^{k / 2}\left|\widetilde{V}_{t, s}\right|^{1 / 2}}{\Gamma(1+k / 2)}
$$

Simultaneous confidence intervals for the $k$ components of $\delta$ can be obtained from the following consequence of the Cauchy-Schwarz inequality: $x \in \mathbb{R}^{k}$ satisfies $x^{\prime} x \leq c^{2}$ if and only if $\left|y^{\prime} x\right|<c\left|y^{\prime} y\right|^{1 / 2}$ for all $y \neq 0$. Thus, with $x=\widetilde{V}_{t, s}^{-1 / 2}(\delta-$ $\left.\widetilde{T}_{t, s}\right)$ and $y=\widetilde{V}_{t, s}^{1 / 2} a$, the above equivalence allows to express

$$
\begin{aligned}
\mathscr{R} & =\left\{\delta \in \mathbb{R}^{k}|| a^{\prime}\left(\delta-\widetilde{T}_{t, s}\right) \mid \leq c_{\gamma}^{1 / 2}\left(a^{\prime} \widetilde{V}_{t, s} a\right)^{1 / 2} \forall a\right. \\
& \neq 0\}
\end{aligned}
$$

and therefore

$$
\begin{aligned}
\mathscr{R} & \subset\left\{\delta \in \mathbb{R}^{k}|| \delta_{i}-\left(\widetilde{T}_{t, s}\right)_{i} \mid \leq c_{\gamma}^{1 / 2}\left(\widetilde{V}_{t, s}\right)_{i, i}^{1 / 2} \forall i\right. \\
& =1, \ldots, k\} .
\end{aligned}
$$

This last set provides simultaneous confidence intervals for the components of $\delta$ with confidence level greater than $1-\gamma$.

4.4. An Example with Simulation Results. In order to evaluate the performance of the proposed confidence region and confidence intervals, we will analyze a particular situation that allows accomplishing simulation studies.

The considered problem is the case of two Wiener processes, $X_{t}$ and $Y_{s}$, with

$$
\begin{aligned}
\mathrm{E}\left[\mathbf{X}_{t}\right] & =A_{1} t^{a} \theta_{1}, \\
\operatorname{Cov}\left(\mathbf{X}_{t}, \mathbf{X}_{t^{\prime}}\right) & =\sigma_{1}^{2} K_{1}\left(t \wedge t^{\prime}\right), \\
\mathrm{E}\left[\mathbf{Y}_{s}\right] & =A_{2} s^{b} \theta_{2}, \\
\operatorname{Cov}\left(\mathbf{Y}_{s}, \mathbf{Y}_{s^{\prime}}\right) & =\sigma_{2}^{2} K_{2}\left(s \wedge s^{\prime}\right) .
\end{aligned}
$$

The estimators $\theta_{t}^{(i)}$ defined in (4) together with their covariance matrices $\sigma_{t}^{(i)}$ have been determined in [8]:

$$
\theta_{t}^{(1)}=\frac{2 a-1}{a t^{2 a-1}}\left(A_{1}^{\prime} K_{1} A_{1}\right)^{-1} A_{1} K_{1}^{-1} \int_{0}^{t} u^{a-1} d X_{u}
$$


while

$$
\left(\sigma_{t}^{(1)}\right)^{-1}=\frac{a^{2} t^{2 a-1}}{2 a-1} A_{1}^{\prime} K_{1}^{-1} A_{1}
$$

Similar results hold for $\theta_{s}^{(2)}$ and $\sigma_{s}^{(2)}$. We will take $a=b=1$, so that

$$
\begin{aligned}
& \sigma_{t}^{(1)}=t^{-1}\left(A_{1}^{\prime} K_{1}^{-1} A_{1}\right)^{-1}, \\
& \sigma_{t}^{(2)}=s^{-1}\left(A_{2}^{\prime} K_{2}^{-1} A_{2}\right)^{-1} .
\end{aligned}
$$

Since $\left(A_{1}^{\prime} K_{1}^{-1} A_{1}\right)^{-1}$ and $\left(A_{2}^{\prime} K_{2}^{-1} A_{2}\right)^{-1}$ are positive definite symmetric matrices, there exists a nonsingular $k \times k$ matrix $L$ that simultaneously diagonalizes them:

$$
\begin{aligned}
& L^{\prime}\left(A_{1}^{\prime} K_{1}^{-1} A_{1}\right)^{-1} L=I, \\
& L^{\prime}\left(A_{2}^{\prime} K_{2}^{-1} A_{2}\right)^{-1} L=\Lambda=\operatorname{diag}\left(\lambda_{1}, \ldots, \lambda_{k}\right)
\end{aligned}
$$

and therefore $L^{\prime} \widetilde{V}_{t, s} L=\widetilde{\eta}_{1} t^{-1} I+\widetilde{\eta}_{2} s^{-1} \Lambda$.

The random variable $T_{t, s}^{\star}=L^{\prime} T_{t, s}$ has distribution $N_{k}\left(\delta^{\star}, \sigma_{1}^{2} t^{-1} I+\sigma_{2}^{2} s^{-1} \Lambda\right)$ with $\delta^{\star}=L^{\prime} \delta$ and the confidence region (26) may be written as

$$
\begin{aligned}
\mathscr{R} & =\{\delta \\
& \in \mathbb{R}^{k} \mid\left(\delta^{\star}-\widetilde{T}_{t, s}^{\star}\right)^{\prime}\left(\widetilde{\eta}_{1} t^{-1} I+\widetilde{\eta}_{2} s^{-1} \Lambda\right)^{-1}\left(\delta^{\star}-\widetilde{T}_{t, s}^{\star}\right) \\
& \left.\leq c_{\gamma}\right\}=L^{\prime-1}\left\{\delta^{\star}\right. \\
& \in \mathbb{R}^{k} \mid\left(\delta^{\star}-\widetilde{T}_{t, s}^{\star}\right)^{\prime}\left(\widetilde{\eta}_{1} t^{-1} I+\widetilde{\eta}_{2} s^{-1} \Lambda\right)^{-1}\left(\delta^{\star}-\widetilde{T}_{t, s}^{\star}\right) \\
& \left.\leq c_{\gamma}\right\} .
\end{aligned}
$$

Thus, the basic case corresponds to $\sigma_{t}^{(1)}=t^{-1} I$ and $\sigma_{s}^{(2)}=$ $s^{-1} \Lambda$, from which the confidence region for more general cases may be constructed. With this simple choice, the matrix $M_{1}$ in (19) is the diagonal matrix

$$
M_{1}=\operatorname{diag}\left(\frac{s \widetilde{\eta}_{1}}{s \widetilde{\eta}_{1}+t \widetilde{\eta}_{2} \lambda_{1}}, \ldots, \frac{s \widetilde{\eta}_{1}}{s \widetilde{\eta}_{1}+t \widetilde{\eta}_{2} \lambda_{k}}\right)
$$

and (20) may be written as

$$
\begin{aligned}
& W_{t, s} \\
& \qquad=\frac{1}{2 k} \sum_{i=1}^{k} Z_{i}^{2}\left(\frac{1}{\beta} \frac{s \widetilde{\eta}_{1}}{s \widetilde{\eta}_{1}+t \widetilde{\eta}_{2} \lambda_{i}}+\frac{1}{1-\beta} \frac{t \widetilde{\eta}_{2} \lambda_{i}}{s \widetilde{\eta}_{1}+t \widetilde{\eta}_{2} \lambda_{i}}\right) .
\end{aligned}
$$

In the following algorithms we suppose that the values of the dimension $k$ and the variance parameters: $\sigma_{1}^{2}, \sigma_{2}^{2}$, and $\lambda_{1}, \ldots, \lambda_{k}$ are given. The values of $t, s$, and $\gamma$ are also fixed.
TABLE 1: Sample percentiles and its validation.

\begin{tabular}{lcc}
\hline $1-\gamma$ & $c_{\gamma}$ & $1-\gamma^{\prime}$ \\
\hline 0.95 & 2.7182 & 0.97 \\
0.90 & 2.3249 & 0.935 \\
0.85 & 1.8182 & 0.848 \\
\hline
\end{tabular}

Algorithm 5. (1) Generate the independent variables $u_{1}, u_{2}$ with a $\chi_{k}^{2}$ distribution.

(2) Compute $\widetilde{\eta}_{1}=\sigma_{1}^{2} u_{1}$ and $\widetilde{\eta}_{2}=\sigma_{2}^{2} u_{2}$, and subsequently, for each $i=1, \ldots, k$,

$$
\begin{aligned}
C_{i} & =\frac{s \widetilde{\eta}_{1}}{s \widetilde{\eta}_{1}+t \widetilde{\eta}_{2} \lambda_{i}}, \\
D_{i} & =\frac{t \widetilde{\eta}_{2} \lambda_{i}}{s \widetilde{\eta}_{1}+t \widetilde{\eta}_{2} \lambda_{i}} .
\end{aligned}
$$

(3) Fixing a large number of iterations $m$, for each $j=$ $1, \ldots, m$,

(a) generate $Z_{j}$ with a $k$-variate $t$-distribution with $2 k$ degrees of freedom;

(b) generate $\beta_{j}$ with a $\operatorname{Beta}(k / 2, k / 2)$ distribution;

(c) compute

$$
W_{j}=\frac{1}{2 k} \sum_{i=1}^{k} Z_{j, i}^{2}\left(\frac{C_{i}}{\beta_{j}}+\frac{D_{i}}{1-\beta_{j}}\right) .
$$

(4) Determine $c_{\gamma}$ as the $1-\gamma$ percentile of the sample $\left(W_{j}\right)_{j=1}^{m}$.

The obtained value of $c_{\gamma}$ may be validated by generating a new sample $\left(W_{j}^{\prime}\right)_{j=1}^{m^{\prime}}$ in which the proportion $1-\gamma^{\prime}$ of $\left(W_{j}^{\prime}\right)$ less than $c_{\gamma}$ may be estimated. Table 1 shows the results obtained by means of a MATLAB program, using the data $k=10, \sigma_{1}^{2}=$ $2, \sigma_{2}^{2}=4, \lambda_{i}=1 / i$ for $i=1, \ldots, 10, t=25, s=50$, and $m=m^{\prime}=1000$.

The next algorithm is designed to obtain the expected volume of the generalized confidence region and the coverage probability of a given vector $\boldsymbol{\delta}=\left(\delta_{1}, \ldots, \delta_{k}\right)$.

Algorithm 6. Given a large number of iterations $m$, for each $j=1, \ldots, m$,

(1) generate a $k$-dimensional vector $\widetilde{T}_{j}$ with independent components, such that $\widetilde{T}_{j, i}$ has a distribution $N\left(\delta_{i}, \sigma_{1}^{2} / t+\sigma_{2}^{2} \lambda_{i} / s\right)$ for each $i=1, \ldots, k$;

(2) generate $\widetilde{\eta}_{1, j}$ and $\widetilde{\eta}_{2, j}$ as in Algorithm 5;

(3) compute

$$
W_{j}=\sum_{i=1}^{k}\left(\delta_{i}-\widetilde{T}_{j, i}\right)^{2} \frac{t s}{\tilde{\eta}_{1, j} s+\widetilde{\eta}_{2, j} \lambda_{i} t},
$$

where the coefficients are the terms of the diagonal matrix $\left(\widetilde{\eta}_{1} t^{-1} I+\widetilde{\eta}_{2} s^{-1} \Lambda\right)^{-1}$; 
TABLE 2: Coverage probability and expected volume of the confidence region.

\begin{tabular}{lcc}
\hline $1-\gamma$ & $p_{c}(\boldsymbol{\delta})$ & $\bar{v}$ \\
\hline 0.95 & 0.982 & 271.85 \\
0.90 & 0.947 & 130.45 \\
0.85 & 0.891 & 37.30 \\
\hline
\end{tabular}

(4) use the value $c_{\gamma}$ given in Algorithm 5 to compute the estimated coverage probability of $\boldsymbol{\delta}$ as the proportion $p_{c}(\boldsymbol{\delta})$ of $W_{j}$ less than $c_{\gamma}$;

(5) according to (27) compute the volume

$$
v_{j}=\frac{\pi^{k / 2} c_{\gamma}^{k / 2}}{\Gamma(1+k / 2)}\left(\prod_{i=1}^{k}\left(\tilde{\eta}_{1, j} t^{-1}+\tilde{\eta}_{2, j} s^{-1} \lambda_{i}\right)\right)^{1 / 2} ;
$$

and the estimated expected volume $\bar{v}=(1 / m) \sum_{j=1}^{m} v_{j}$.

Taking for instance $\boldsymbol{\delta}=(1,-1,2,0,3,4,-2 ., 5,-3,1)$ and with the remaining data as before, the obtained coverage probability and estimated expected volume are shown in Table 2.

Finally an algorithm may be designed in order to simulate the simultaneous confidence intervals for the components of $\boldsymbol{\delta}$ and to estimate the joint coverage probability.

Algorithm 7. Given a large number of iterations $m$, for each $j=1, \ldots, m$ the following hold.

(1) For each $i=1, \ldots, k$, compute, according to the results of the previous algorithms, the extremes of the intervals $I_{j, i}=\left(l_{j, i}, L_{j, i}\right)$, where

$$
\begin{aligned}
l_{j, i} & =\widetilde{T}_{j, i}-c_{\gamma}^{1 / 2}\left(\frac{\tilde{\eta}_{1, j}}{t}+\frac{\tilde{\eta}_{2, j} \lambda_{i}}{s}\right)^{1 / 2}, \\
L_{j, i} & =\widetilde{T}_{j, i}+c_{\gamma}^{1 / 2}\left(\frac{\tilde{\eta}_{1, j}}{t}+\frac{\tilde{\eta}_{2, j} \lambda_{i}}{s}\right)^{1 / 2} .
\end{aligned}
$$

(2) Compute the estimated joint coverage probability of $\boldsymbol{\delta}$ as the proportion $p_{c}(\boldsymbol{\delta})$ of iterations such that $\delta_{i} \in I_{j, i}$ for all $i=1, \ldots, k$.

(3) Compute

$$
\begin{gathered}
l_{i}=\frac{1}{m} \sum_{j=1}^{m} l_{j, i}, \\
L_{i}=\frac{1}{m} \sum_{j=1}^{m} L_{j, i}
\end{gathered}
$$

that gives the expected length $L_{i}-l_{i}$ of the interval $\bar{I}_{i}=\left(l_{i}, L_{i}\right)$.

With the same data as before and the same vector $\boldsymbol{\delta}$, the obtained results are shown in Table 3. Let us observe that the coverage probability of the generalized confidence region $p_{c}(\boldsymbol{\delta})$ always exceeds the confidence level and that the coverage probability of the confidence intervals is always very close to 1 . Taking alternative parameter values, some other simulations have been made with similar results.

\section{Inferences about the Vector Means of Several Independent Gaussian Processes}

As a generalization we now consider the case of $N$ independent multivariate Gaussian processes $X_{t_{1}}^{(1)}, X_{t_{2}}^{(2)}, \ldots, X_{t_{N}}^{(N)}$ with dimensions $p_{1}, p_{2}, \ldots, p_{N}$ and time parameters $t_{1}, t_{2}$, $\ldots, t_{N}$ varying in some interval $T$. We assume, for each $i=$ $1, \ldots, N$, the following:

$$
\begin{gathered}
\mathrm{E}_{\theta_{i}}\left[X_{t_{i}}^{(i)}\right]=A_{i}\left(t_{i}\right) \theta_{i}, \\
\operatorname{Cov}\left(X_{t_{i}}^{(i)}, X_{t_{i}^{\prime}}^{(i)}\right)=\sigma_{i}^{2} K\left(t_{i}, t_{i}^{\prime}\right),
\end{gathered}
$$

where $\theta_{1}, \ldots, \theta_{N} \in \mathbb{R}^{k}$ and $\sigma_{1}^{2}, \ldots, \sigma_{N}^{2} \in \mathbb{R}$ are unknown parameters.

5.1. Testing the Equality of Means. We first consider a test of the null hypothesis $H_{0}: \theta_{1}=\theta_{2}=\cdots=\theta_{N}$, or $H_{0}: \boldsymbol{\delta}=\mathbf{0}$ with $\delta_{i}=\theta_{i}-\theta_{N}$ for $i=1,2, \ldots, N-1$.

According to the method of Section 3, we may consider the estimators $\theta_{t_{i}}^{(i)}$ of the parameters $\theta_{i}$, whose distribution is $N_{k}\left(\theta_{i}, \sigma_{i}^{2} \sigma_{t_{i}}^{(i)}\right)$, and also the differences $T_{t_{i}, t_{N}}^{(i)}=\theta_{t_{i}}^{(i)}-\theta_{t_{N}}^{(N)}$ for $i=1,2, \ldots, N-1$ with distributions $N_{k}\left(\delta_{i}, \sigma_{i}^{2} \sigma_{t_{i}}^{(i)}+\sigma_{N}^{2} \sigma_{t_{N}}^{(N)}\right)$.

The $(N-1) k$-dimensional vector

$$
T_{\mathbf{t}}^{\prime}=T_{t_{1}, \ldots, t_{N}}^{\prime}=\left(T_{t_{1}, t_{N}}^{(1)}, T_{t_{2}, t_{N}}^{(2)}, \ldots, T_{t_{N-1}, t_{N}}^{(N-1)}\right)
$$

will have a multivariate normal distribution with vector mean $\boldsymbol{\delta}$ and covariance matrix

$$
U_{\mathbf{t}}=\left(\begin{array}{llll}
\sigma_{1}^{2} \sigma_{t_{1}}^{(1)} & & & \\
& \sigma_{2}^{2} \sigma_{t_{2}}^{(2)} & & \\
& & \ddots & \\
& & & \sigma_{N-1}^{2} \sigma_{t_{N-1}}^{(N-1)}
\end{array}\right)
$$

$$
\begin{gathered}
+\left(\begin{array}{cccc}
\sigma_{N}^{2} \sigma_{t_{N}}^{(N)} & \sigma_{N}^{2} \sigma_{t_{N}}^{(N)} & \ldots & \sigma_{N}^{2} \sigma_{t_{N}}^{(N)} \\
\sigma_{N}^{2} \sigma_{t_{N}}^{(N)} & \sigma_{N}^{2} \sigma_{t_{N}}^{(N)} & \ldots & \sigma_{N}^{2} \sigma_{t_{N}}^{(N)} \\
\vdots & \vdots & \ddots & \vdots \\
\sigma_{N}^{2} \sigma_{t_{N}}^{(N)} & \sigma_{N}^{2} \sigma_{t_{N}}^{(N)} & \ldots & \sigma_{N}^{2} \sigma_{t_{N}}^{(N)}
\end{array}\right) \\
=\operatorname{diag}\left(\sigma_{1}^{2} \sigma_{t_{1}}^{(1)}, \ldots, \sigma_{N-1}^{2} \sigma_{t_{N-1}}^{(N-1)}\right)+\mathbf{J} \sigma_{N}^{2} \sigma_{t_{N}}^{(N)} \mathbf{J}^{\prime},
\end{gathered}
$$

where $\mathbf{J}$ is the Kronecker product $\mathbf{1}_{N-1} \otimes I_{k}$ (composed by a column of $N-1$ copies of the identity matrix $I_{k}$ ). 
TABLE 3: Confidence intervals and joint coverage probabilities.

\begin{tabular}{|c|c|c|c|c|c|}
\hline \multicolumn{2}{|c|}{$1-\gamma=0.95$} & \multicolumn{2}{|c|}{$1-\gamma=0.90$} & \multicolumn{2}{|c|}{$1-\gamma=0.85$} \\
\hline $\bar{I}_{i}$ & $L_{i}-l_{i}$ & $\bar{I}_{i}$ & $L_{i}-l_{i}$ & $\bar{I}_{i}$ & $L_{i}-l_{i}$ \\
\hline$(-1.29,3.32)$ & 4.61 & $(-1.09,3.14)$ & 4.23 & $(-0.88,2.89)$ & 3.73 \\
\hline$(-2.79,0.79)$ & 3.58 & $(-2.64,0.65)$ & 3.29 & $(-2.46,0.44)$ & 2.90 \\
\hline$(0.41,3.57)$ & 3.16 & $(0.56,3.46)$ & 2.90 & $(0.72,3.28)$ & 2.56 \\
\hline$(-1.47,1.45)$ & 2.92 & $(-1.34,1.35)$ & 2.69 & $(-1.19,1.18)$ & 2.37 \\
\hline$(1.60,4.36)$ & 2.76 & $(1.73,4.28)$ & 2.55 & $(1.87,4.13)$ & 2.25 \\
\hline$(2.67,5.33)$ & 2.66 & $(2.77,5.22)$ & 2.45 & $(2.93,5.09)$ & 2.16 \\
\hline$(-3.29,-0.71)$ & 2.58 & $(-3.19,-0.81)$ & 2.38 & $(-3.05,-0.95)$ & 2.10 \\
\hline$(3.73,6.25)$ & 2.52 & $(3.83,6.16)$ & 2.33 & $(3.99,6.04)$ & 2.05 \\
\hline$(-4.24,-1.77)$ & 2.47 & $(-4.14,-1.86)$ & 2.28 & $(-4.01,-1.99)$ & 2.01 \\
\hline$(-0.21,2.22)$ & 2.43 & $(-0.12,2.12)$ & 2.24 & $(0.01,1.99)$ & 1.98 \\
\hline \multicolumn{2}{|c|}{$p_{c}(\boldsymbol{\delta})=1$} & \multicolumn{2}{|c|}{$p_{c}(\boldsymbol{\delta})=0.9997$} & $p_{c}(\boldsymbol{\delta}$ & \\
\hline
\end{tabular}

For each $i=1, \ldots, N$, given $t_{i, 1}<t_{i, 2}<t_{i}$ such that $\sigma_{t_{i, 1}}^{(i)}-$ $\sigma_{t_{i}, 2}^{(i)}$ is nonsingular, let

$$
\eta_{i}=\left(\theta_{t_{i, 2}}^{(i)}-\theta_{t_{i, 1}}^{(i)}\right)^{\prime}\left(\sigma_{t_{i, 1}}^{(i)}-\sigma_{t_{i, 2}}^{(i)}\right)^{-1}\left(\theta_{t_{i, 2}}^{(i)}-\theta_{t_{i, 1}}^{(i)}\right)
$$

which are independent random variables, independent also of $T_{\mathbf{t}}$ and such that $u_{i}=\eta_{i} / \sigma_{i}^{2}$ has $\chi_{k}^{2}$ distribution.

The covariance matrix $U_{t}$ may be estimated by means of

$$
V_{\mathbf{t}}=\operatorname{diag}\left(\eta_{1} \sigma_{t_{1}}^{(1)}, \ldots, \eta_{N-1} \sigma_{t_{N-1}}^{N-1}\right)+\mathbf{J} \eta_{N} \sigma_{t_{N}}^{(N)} \mathbf{J}^{\prime}
$$

with observed value

$$
\widetilde{V}_{\mathbf{t}}=\operatorname{diag}\left(\widetilde{\eta}_{1} \sigma_{t_{1}}^{(1)}, \ldots, \widetilde{\eta}_{N-1} \sigma_{t_{N-1}}^{(1)}\right)+\mathbf{J} \widetilde{\eta}_{N} \sigma_{t_{N}}^{(N)} \mathbf{J}^{\prime} .
$$

As in (17), the matrix

$$
\begin{aligned}
R_{\mathbf{t}} & =\widetilde{V}_{\mathbf{t}}^{-1 / 2}\left[\operatorname{diag}\left(\sigma_{1}^{2} \sigma_{t_{1}}^{(1)} \frac{\tilde{\eta}_{1}}{\eta_{1}}, \ldots, \sigma_{N-1}^{2} \sigma_{t_{N-1}}^{(N-1)} \frac{\widetilde{\eta}_{N-1}}{\eta_{N-1}}\right)\right. \\
& \left.+\mathbf{J} \sigma_{N}^{2} \sigma_{t_{N}}^{(N)} \frac{\widetilde{\eta}_{N}}{\eta_{N}} \mathbf{J}^{\prime}\right] \widetilde{V}_{\mathbf{t}}^{-1 / 2} \\
& =\widetilde{V}_{\mathbf{t}}^{-1 / 2}\left[\operatorname{diag}\left(\sigma_{t_{1}}^{(1)} \frac{\widetilde{\eta}_{1}}{u_{1}}, \ldots, \sigma_{t_{N-1}}^{(N-1)} \frac{\widetilde{\eta}_{N-1}}{u_{N-1}}\right)\right. \\
& \left.+\mathbf{J} \sigma_{t_{N}}^{(N)} \frac{\widetilde{\eta}_{N}}{u_{N}} \mathbf{J}^{\prime}\right] \widetilde{V}_{\mathbf{t}}^{-1 / 2}
\end{aligned}
$$

does not depend on any parameter and is independent of $T_{\mathbf{t}}$; moreover its observed value is

$$
\widetilde{R}_{\mathbf{t}}=\widetilde{V}_{\mathbf{t}}^{-1 / 2} U_{\mathbf{t}} \widetilde{V}_{\mathbf{t}}^{-1 / 2}
$$

Let us define

$$
\begin{aligned}
& d_{\mathbf{t}}=\widetilde{V}_{\mathbf{t}}^{-1 / 2} T_{\mathbf{t}}, \\
& Z_{\mathbf{t}}=\widetilde{R}_{\mathbf{t}}^{-1 / 2} d_{\mathbf{t}}
\end{aligned}
$$

that have, under $H_{0}$, distributions $N_{(N-1) k}\left(0, \widetilde{R}_{\mathbf{t}}\right)$ and $N_{(N-1) k}(0, I)$, respectively. So that, finally,

$$
W_{\mathbf{t}}=Z_{\mathbf{t}}^{\prime} R_{\mathbf{t}} Z_{\mathbf{t}} \text { and its observed value } \widetilde{W}_{\mathbf{t}}=\widetilde{T}_{\mathbf{t}}^{\prime} \widetilde{V}_{\mathbf{t}}^{-1} \widetilde{T}_{\mathbf{t}}
$$

are scalars not depending on the parameters and the distribution of $W_{\mathbf{t}}$ neither depends on $\left(\sigma_{1}^{2}, \ldots, \sigma_{N}^{2}\right)$. Moreover, $W_{\mathbf{t}}$ is a positive definite quadratic form in $Z_{\mathbf{t}}$ whose distribution under $H_{1}$ is $N_{(N-1) k}(\overline{\boldsymbol{\delta}}, I)$ with $\overline{\boldsymbol{\delta}}=\left(\widetilde{V}_{\mathbf{t}}^{-1 / 2} U_{\mathbf{t}} \widetilde{V}_{\mathbf{t}}^{-1 / 2}\right)^{-1 / 2} \widetilde{V}_{\mathbf{t}}^{-1 / 2} \boldsymbol{\delta}$. Hence $W_{\mathbf{t}}$ is stochastically larger under $H_{1}$ than under $H_{0}$ and we get the following.

Proposition 8. $W_{\mathrm{t}}$ is a generalized test process for testing $H_{0}$ : $\boldsymbol{\delta}=\mathbf{0}$ against $H_{1}: \boldsymbol{\delta} \neq \mathbf{0}$.

An alternative expression will be more suitable for the calculation of the $p$ values. For each $i=1, \ldots, N-1$, let $G_{i}$ be the $(N-1) k \times(N-1) k$ square matrix obtained by replacing in the matrix (46) the $i$ block with $\widetilde{\eta}_{i} \sigma_{t_{i}}^{(i)}$ while the remaining are replaced with $\mathbf{0}$ (of order $k \times k$ ); that means

$$
G_{i}=G_{i}\left(t_{i}\right)=\operatorname{diag}\left(\mathbf{0}, \ldots, \mathbf{0}, \tilde{\eta}_{i} \sigma_{t_{i}}^{(i)}, \mathbf{0}, \ldots, \mathbf{0}\right) .
$$

Now

$$
\begin{aligned}
M_{i} & =\widetilde{V}_{\mathbf{t}}^{-1 / 2} G_{i} \widetilde{V}_{\mathbf{t}}^{-1 / 2}, \\
M_{N} & =\widetilde{V}_{\mathbf{t}}^{-1 / 2} \mathbf{J}{\widetilde{\eta_{N}}}_{t_{N}}^{(N)} \mathbf{J}^{\prime} \widetilde{V}_{\mathbf{t}}^{-1 / 2}
\end{aligned}
$$

satisfy $\sum_{i=1}^{N-1} M_{i}+M_{N}=I$ and allow expressing

$$
R_{\mathbf{t}}=\sum_{i=1}^{N-1} \frac{M_{i}}{u_{i}}+\frac{M_{N}}{u_{N}}=\sum_{i=1}^{N-1} \frac{M_{i}}{u_{i}}+\frac{I-\sum_{i=1}^{N-1} M_{i}}{u_{N}} .
$$

Consequently

$$
\begin{aligned}
W_{\mathbf{t}} & =Z_{\mathbf{t}}^{\prime}\left(\sum_{i=1}^{N-1} \frac{M_{i}}{u_{i}}+\frac{I-\sum_{i=1}^{N-1} M_{i}}{u_{N}}\right) Z_{\mathbf{t}} \\
& =\frac{1}{N k} \bar{Z}_{\mathbf{t}}^{\prime}\left(\sum_{i=1}^{N-1} \frac{M_{i}}{\beta_{i}}+\frac{I-\sum_{i=1}^{N-1} M_{i}}{1-\sum_{i=1}^{N-1} \beta_{i}}\right) \bar{Z}_{\mathbf{t}},
\end{aligned}
$$


where

$$
\bar{Z}_{\mathbf{t}}=\frac{\sqrt{N k}}{\left(\sum_{i=1}^{N} u_{i}\right)^{1 / 2}} Z_{\mathbf{t}}
$$

has a $(N-1) k$-multivariate Student $t$-distribution, with $N k$ degrees of freedom, while $\beta_{i}=u_{i} /\left(\sum_{i=1}^{N-1} u_{i}\right)$ is such that $\left(\beta_{1}, \ldots, \beta_{N-1}\right)$ has a Dirichlet distribution with parameters $(k / 2, \ldots, k / 2)$ and is independent of $\bar{Z}_{\mathbf{t}}$.

After the observation of $M_{1}, \ldots, M_{N-1}$, the generalized $p$ value of the given test is

$$
\begin{aligned}
& p_{\mathbf{t}}\left(M_{1}, \ldots, M_{N-1}\right) \\
& \quad=P_{\boldsymbol{\delta}=\mathbf{0}}\left\{\frac{1}{N k} \bar{Z}_{\mathbf{t}}^{\prime}\left(\sum_{i=1}^{N-1} \frac{M_{i}}{\beta_{i}}+\frac{I-\sum_{i=1}^{N-1} M_{i}}{1-\sum_{i=1}^{N-1} \beta_{i}}\right) \bar{Z}_{\mathbf{t}}\right. \\
& \left.\quad \geq \widetilde{W_{\mathbf{t}}}\right\} .
\end{aligned}
$$

As in Section 4.2, an analogous property of invariance of the test statistic $W_{\mathbf{t}}$ may be stated with respect to the transformations of each process

$$
X_{t_{i}}^{(i)^{\star}}=Q_{i} X_{t_{i}}^{(i)}
$$

for nonsingular matrices $Q_{i}$.

5.2. Confidence Region for $\boldsymbol{\delta}$. For any value of the unknown parameter $\boldsymbol{\delta}$, the difference $T_{\mathbf{t}}^{\boldsymbol{\delta}}=T_{\mathbf{t}}-\boldsymbol{\delta}$ has distribution $N_{(N-1) k}\left(0, U_{\mathbf{t}}\right)$ and $Z_{\mathbf{t}}^{\delta}=\left(\widetilde{V}_{\mathbf{t}}^{-1 / 2} U_{\mathbf{t}} \widetilde{V}_{\mathbf{t}}^{-1 / 2}\right)^{-1 / 2} \widetilde{V}_{\mathbf{t}}^{-1 / 2} T_{\mathbf{t}}^{\delta}$ is $N_{(N-1) k}(0, I)$. Hence

$$
W_{\mathbf{t}}^{\boldsymbol{\delta}}=\left(Z_{\mathbf{t}}^{\boldsymbol{\delta}}\right)^{\prime} R_{\mathbf{t}} Z_{\mathbf{t}}^{\boldsymbol{\delta}}
$$

has the same distribution as $W_{\mathbf{t}}$ under $H_{0}: \boldsymbol{\delta}=\mathbf{0}$, which is independent of all the parameters, while its observed value $\widetilde{W}_{\mathbf{t}}^{\delta}$ does not depend on the nuisance parameters. The following proposition is thus established.

Proposition 9. $W_{\mathbf{t}}^{\delta}$ is a generalized pivotal quantity and a generalized confidence region for $\boldsymbol{\delta}$ is given by $\left\{\boldsymbol{\delta} \in \mathbb{R}^{(N-1) k}\right.$ $\left.\widetilde{W}_{\mathbf{t}}^{\delta} \leq c_{\gamma}\right\}$, if $P\left\{W_{\mathbf{t}}^{\delta} \leq c_{\gamma}\right\}=1-\gamma$.

As observed in Section 4.3, the just obtained confidence region may be written as

$$
\begin{aligned}
\mathscr{R} & =\left\{\boldsymbol{\delta} \in \mathbb{R}^{(N-1) k}|| a^{\prime}\left(\widetilde{T}_{\mathbf{t}}-\boldsymbol{\delta}\right) \mid\right. \\
& \left.\leq c_{\gamma}^{1 / 2}\left(a^{\prime} \widetilde{V}_{\mathbf{t}} a\right)^{1 / 2} \forall a \in \mathbb{R}^{(N-1) k}, a \neq 0\right\} .
\end{aligned}
$$

For $j \leq N-1$ and $i \leq k$, let $a_{j, i}$ represent the vector in $\mathbb{R}^{(N-1) k}$ whose only nonzero term is 1 in the $(j-1) k+i$ position, whereas $a_{j, m, i}=a_{j, i}-a_{m, i}$ for $j<m \leq N-1$. Then

$$
\begin{aligned}
a_{j, i}^{\prime} \boldsymbol{\delta} & =\theta_{i}^{(j)}-\theta_{i}^{(N)}, \\
a_{j, i}^{\prime} \widetilde{T}_{\mathbf{t}} & =\left(\widetilde{\theta}_{t_{j}}^{(j)}-\widetilde{\theta}_{t_{N}}^{(N)}\right)_{i},
\end{aligned}
$$

$$
\begin{aligned}
a_{j, m, i}^{\prime} \boldsymbol{\delta} & =\theta_{i}^{(j)}-\theta_{i}^{(m)}, \\
a_{j, m, i}^{\prime} \widetilde{T}_{\mathbf{t}} & =\left(\widetilde{\theta}_{t_{j}}^{(j)}-\widetilde{\theta}_{t_{m}}^{(m)}\right)_{i} .
\end{aligned}
$$

And from (51) we get

$$
\begin{aligned}
a_{j, i}^{\prime} \widetilde{V}_{\mathbf{t}} a_{j, i} & =\widetilde{\eta}_{j}\left(\sigma_{t_{j}}^{(j)}\right)_{i, i}+\widetilde{\eta}_{N}\left(\sigma_{t_{N}}^{(N)}\right)_{i, i}, \\
a_{j, m, i}^{\prime} \widetilde{V}_{\mathbf{t}} a_{j, m, i} & =\widetilde{\eta}_{j}\left(\sigma_{t_{j}}^{(j)}\right)_{i, i}+\widetilde{\eta}_{m}\left(\sigma_{t_{m}}^{(m)}\right)_{i, i} .
\end{aligned}
$$

Therefore, as in Section 4.3, $\mathscr{R}$ is contained in the intersection of all the intervals:

$$
\begin{array}{r}
\left\{\left|\left(\theta_{j}-\theta_{m}\right)_{i}-\left(\widetilde{\theta}_{t_{j}}^{(j)}-\widetilde{\theta}_{t_{m}}^{(m)}\right)_{i}\right|\right. \\
\left.\leq c_{\gamma}^{1 / 2}\left[\widetilde{\eta}_{j} \sigma_{t_{j}}^{(j)}+\widetilde{\eta}_{m} \sigma_{t_{m}}^{(m)}\right]_{i, i}\right\}
\end{array}
$$

for $1 \leq j<m \leq N$ and $1 \leq i \leq k$. This gives a set of simultaneous confidence intervals for all the components of the differences between the mean parameters of the $N$ processes.

5.3. General Linear Hypotheses. When the null hypothesis $H_{0}: \theta_{1}=\theta_{2}=\cdots=\theta_{N}$ is rejected, one can focus on testing a linear relation between the mean parameters. That is, if $\boldsymbol{\theta}^{\prime}=\left(\theta_{1}^{\prime}, \ldots, \theta_{N}^{\prime}\right), \mathbf{L}$ is an $r \times N k$ matrix, and $\mathbf{l}$ is a vector in $\mathbb{R}^{r}$, the objective is to test the null hypothesis $H_{0}^{\prime}: \mathbf{L} \boldsymbol{\theta}=\mathbf{1}$ against the alternative $H_{1}^{\prime}: \mathbf{L} \boldsymbol{\theta} \neq \mathbf{l}$.

We denote $\boldsymbol{\theta}_{\mathbf{t}}^{\prime}=\left(\theta_{t_{1}}^{(1)^{\prime}}, \ldots, \theta_{t_{N}}^{(N)^{\prime}}\right)$ which has distribution $N_{N k}\left(\boldsymbol{\theta}, \mathbf{U}_{\mathbf{t}}\right)$, where $\mathbf{U}_{\mathbf{t}}=\operatorname{diag}\left(\sigma_{1}^{2} \sigma_{t_{1}}^{(1)}, \ldots, \sigma_{N}^{2} \sigma_{t_{N}}^{(N)}\right)$. Now, $T_{\mathbf{t}}=$ $\mathbf{L} \boldsymbol{\theta}_{\mathbf{t}}-\mathbf{l}$ has $N_{r}\left(\mathbf{L} \boldsymbol{\theta}-\mathbf{l}, \mathbf{L} \mathbf{U}_{\mathbf{t}} \mathbf{L}^{\prime}\right)$ distribution, whose mean vector is $\mathbf{0}$ under $H_{0}^{\prime}$. Moreover $\mathbf{U}_{\mathbf{t}}$ may be estimated by

$$
\mathbf{V}_{\mathbf{t}}=\mathbf{L} \operatorname{diag}\left(\eta_{1} \sigma_{t_{1}}^{(1)}, \ldots, \eta_{N} \sigma_{t_{N}}^{(N)}\right) \mathbf{L}^{\prime}
$$

which has observed value $\widetilde{\mathbf{V}}_{\mathbf{t}}=\mathbf{L} \operatorname{diag}\left(\widetilde{\eta}_{1} \sigma_{t_{1}}^{(1)}, \ldots, \widetilde{\eta}_{N} \sigma_{t_{N}}^{(N)}\right) \mathbf{L}^{\prime}$. Observing that, under $H_{0}^{\prime}$,

$$
D_{\mathbf{t}}=\widetilde{\mathbf{V}}_{\mathbf{t}}^{-1 / 2} T_{\mathbf{t}}
$$

has distribution $N_{r}\left(\mathbf{0}, \widetilde{\mathbf{V}}_{\mathbf{t}}^{-1 / 2} \mathbf{L} \mathbf{U}_{\mathbf{t}} \mathbf{L}^{\prime} \widetilde{\mathbf{V}}_{\mathbf{t}}^{-1 / 2}\right)$, we get that

$$
Z_{\mathbf{t}}=\left(\widetilde{\mathbf{V}}_{\mathbf{t}}^{-1 / 2} \mathbf{L} \mathbf{U}_{\mathbf{t}} \mathbf{L}^{\prime} \widetilde{\mathbf{V}}_{\mathbf{t}}^{-1 / 2}\right)^{-1 / 2} D_{\mathbf{t}}
$$

has $N_{r}(\mathbf{0}, I)$ distribution. Therefore, if

$$
\begin{aligned}
& R_{\mathbf{t}} \\
& =\widetilde{\mathbf{V}}_{\mathbf{t}}^{-1 / 2} \mathbf{L} \operatorname{diag}\left(\sigma_{1}^{2} \sigma_{t_{1}}^{(1)} \frac{\widetilde{\eta}_{1}}{\eta_{1}}, \ldots, \sigma_{N}^{2} \sigma_{t_{N}}^{(N)} \frac{\widetilde{\eta}_{N}}{\eta_{N}}\right) \mathbf{L}^{\prime} \widetilde{\mathbf{V}}_{\mathbf{t}}^{-1 / 2},
\end{aligned}
$$

then

$$
W_{\mathbf{t}}=Z_{\mathbf{t}}^{\prime} R_{\mathbf{t}} Z_{\mathbf{t}}
$$

is a one-dimensional random variable with distribution, under $H_{0}^{\prime}$, independent of the parameters and whose 
observed value, given by $\widetilde{W}_{\mathbf{t}}=T_{\mathbf{t}}^{\prime} \widetilde{V}_{\mathbf{t}} T_{\mathbf{t}}$, does not depend on the parameters. Since $W_{\mathbf{t}}$ is a positive quadratic form in the normal vector $Z_{\mathbf{t}}$ whose mean under $H_{1}$ is different from 0 , $W_{\mathbf{t}}$ is stochastically larger under $H_{1}$ than under $H_{0}$, so that the following proposition is proved.

Proposition 10. $W_{\mathbf{t}}$ is a generalized test process for the contrast of $H_{0}^{\prime}: \mathbf{L} \boldsymbol{\theta}=\mathbf{l}$ against $H_{1}^{\prime}: \mathbf{L} \boldsymbol{\theta} \neq \mathbf{1}$.

With the same matrix $G_{i}$ defined in (56), one can put

$$
M_{i}=M_{i}\left(t_{i}\right)=\widetilde{V}_{\mathbf{t}}^{-1 / 2} \mathbf{L} G_{i} \mathbf{L}^{\prime} \widetilde{V}_{\mathbf{t}}^{-1 / 2}
$$

that satisfy $\sum_{i=1}^{N} M_{i}=I$. Since

$$
R_{\mathbf{t}}=\sum_{i=1}^{N} \frac{M_{i}}{u_{i}}
$$

where $u_{1}, \ldots, u_{N}$ are mutually independent, $\chi_{k}^{2}$ distributed and independent of $Z_{\mathbf{t}}$, we get

$$
W_{\mathbf{t}}=\frac{1}{N k} \bar{Z}_{\mathbf{t}}^{\prime}\left(\sum_{i=1}^{N-1} \frac{M_{i}}{\beta_{i}}+\frac{I-\sum_{i=1}^{N-1} M_{i}}{1-\sum_{i=1}^{N-1} \beta_{i}}\right) \bar{Z}_{\mathbf{t}}
$$

with $\bar{Z}_{\mathbf{t}}$, given by (60), distributed according to a $(N-1) k$ multivariate Student $t$ distribution and $\beta_{i}=u_{i} /\left(\sum_{i=1}^{N-1} u_{i}\right)$ such that $\left(\beta_{1}, \ldots, \beta_{N-1}\right)$ has a Dirichlet distribution with parameters $(k / 2, \ldots, k / 2)$ and is independent of $\bar{Z}_{\mathbf{t}}$. Thus, the generalized $p$ value of the given test can be calculated as in (61), although the expressions of $M_{i}$ are now different.

\section{Conflict of Interests}

The authors declare that there is no conflict of interests regarding the publication of this paper.

\section{References}

[1] K. Tsui and S. Weerahandi, "Generalized $p$-values in significance testing of hypotheses in the presence of nuisance parameters," Journal of the American Statistical Association, vol. 84, no. 406, pp. 602-607, 1989.

[2] S. Weerahandi, "Generalized confidence intervals," Journal of the American Statistical Association, vol. 88, no. 423, pp. 899905, 1993.

[3] J. Gamage, T. Mathew, and S. Weerahandi, "Generalized pvalues and generalized confidence regions for the multivariate Behrens-Fisher problem and MANOVA," Journal of Multivariate Analysis, vol. 88, no. 1, pp. 177-189, 2004.

[4] S. H. Lin, J. C. Lee, and R. S. Wang, "Generalized inferences on the common mean vector of several multivariate normal populations," Journal of Statistical Planning and Inference, vol. 137, no. 7, pp. 2240-2249, 2007.

[5] L.-W. Xu and S.-G. Wang, "A new generalized p-value for ANOVA under heteroscedasticity," Statistics and Probability Letters, vol. 78, no. 8, pp. 963-969, 2008.

[6] J. T. Zhang, "An approximate hotelling $T^{2}$-test for heteroscedastic one-way MANOVA," Open Journal of Statistics, vol. 2, no. 1, pp. 1-11, 2012.
[7] P. Ibarrola and R. Vélez, "Testing and confidence estimation of the mean of a multidimensional Gaussian process," Statistics, vol. 36, no. 4, pp. 317-327, 2002.

[8] P. Ibarrola and R. Vélez, "On Behrens-Fisher problem for continuous time Gaussian processes," Linear Algebra and Its Applications, vol. 389, no. 1-3, pp. 63-76, 2004. 


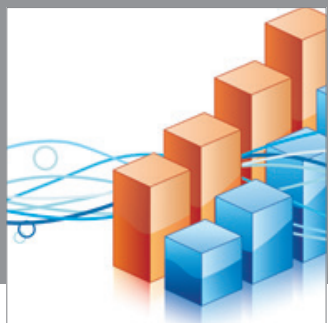

Advances in

Operations Research

mansans

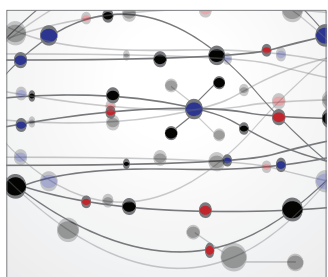

The Scientific World Journal
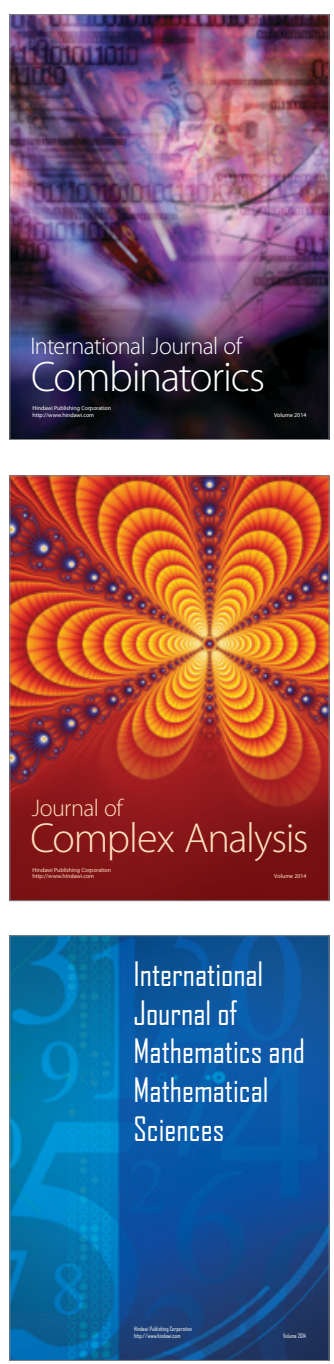
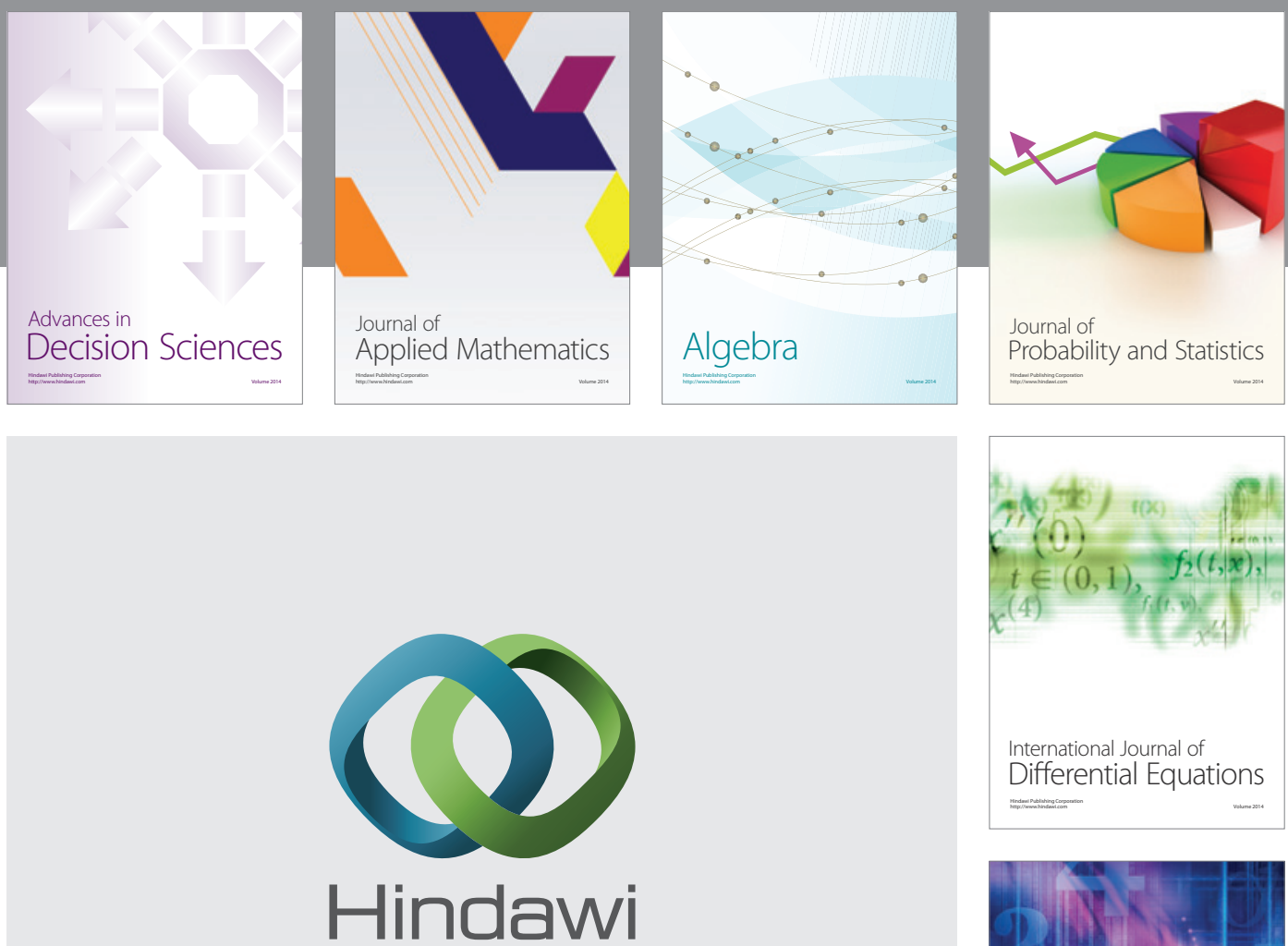

Submit your manuscripts at http://www.hindawi.com
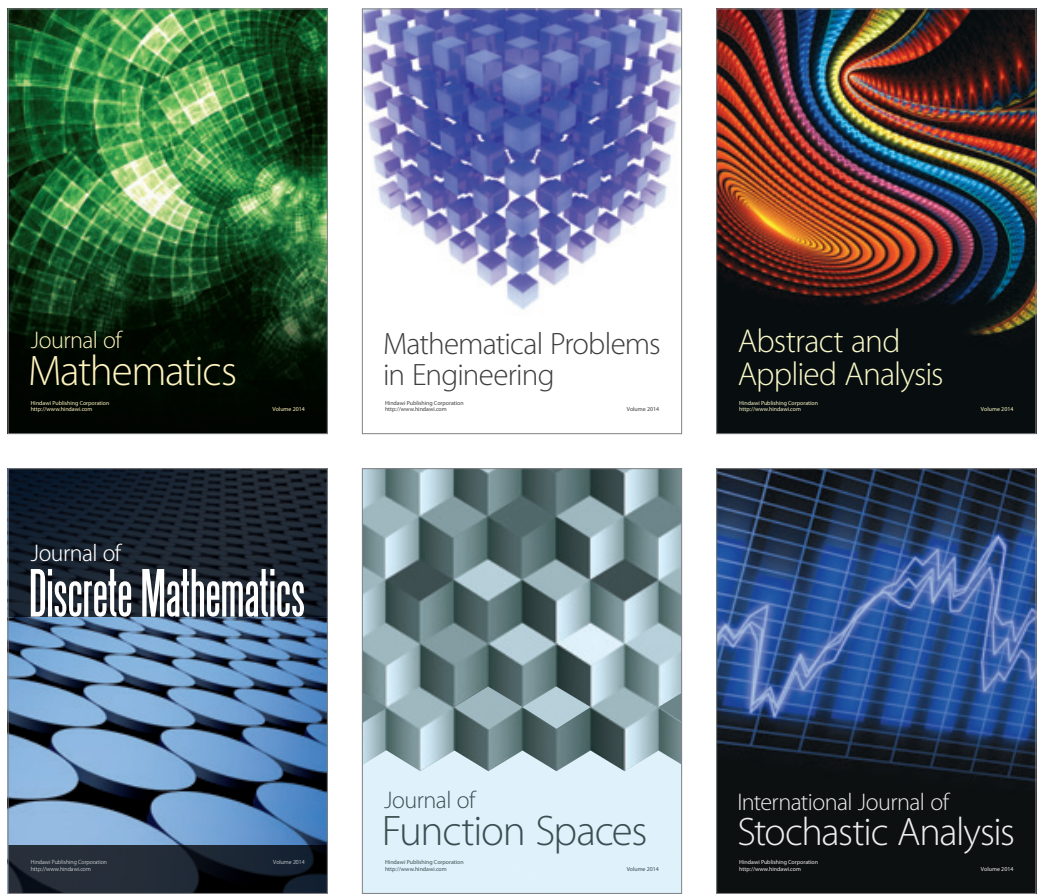

Journal of

Function Spaces

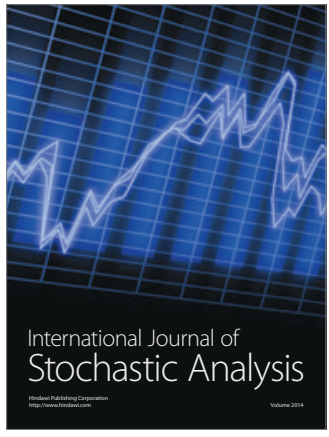

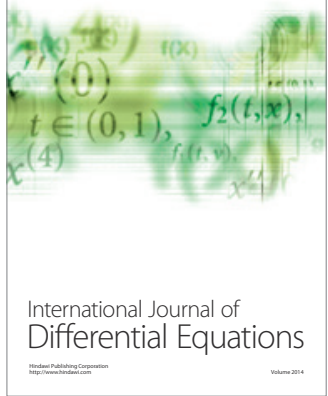
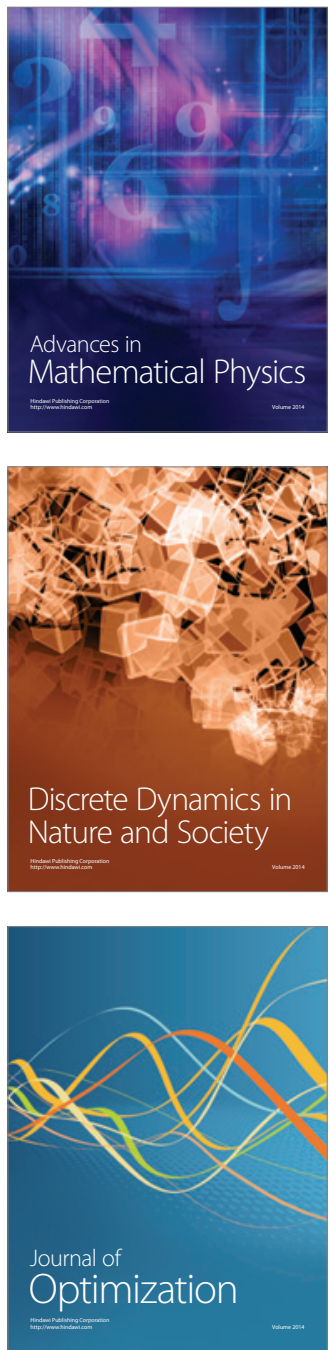